\title{
STIM proteins as regulators of neuronal store-operated calcium influx
}

\author{
Elena Popugaeva*,1 \& Ilya Bezprozvanny ${ }^{1,2}$ \\ ${ }^{1}$ Laboratory of Molecular Neurodegeneration, Department of Medical Physics, Peter the Great St Petersburg Polytechnic University, \\ St Petersburg, 195251, Russia \\ ${ }^{2}$ Department of Physiology, UT Southwestern Medical Center at Dallas, Dallas, TX 75390, USA \\ * Author for correspondence: lena.popugaeva@gmail.com
}

\section{"Both STIM1 and STIM2 proteins are expressed in hippocampal neurons [5], and both of these proteins have been implicated in control of nSOC."}

First draft submitted: 13 November 2017; Accepted for publication: 14 November 2017; Published online: 3 January 2018

\section{Keywords: Alzheimer's disease • hippocampus • nSOC • STIM1 • STIM2}

The store-operated calcium channels (SOC) were initially described in nonexcitable cells, such as $\mathrm{T}$ cells in the immune system [1]. In neurons, the presence of SOC has been controversial [2]. The main argument against neuronal SOC (nSOC) is that neurons possess many other calcium-permeable channels with high conductance, such as NMDA receptors and voltage-gated calcium channels. The existence, functional relevance and molecular identity of nSOC are still under investigation. However, recent molecular analysis of nSOC provided support to its existence and importance for neuronal function.

SOC is mediated by two main components: endoplasmic reticulum (ER) $\mathrm{Ca}^{2+}$ sensors STIM1 or STIM2 proteins [3] and plasma membrane (PM) $\mathrm{Ca}^{2+}$ channels from Orai and TRPC protein families [1]. SOC in nonexcitable cells is activated by $\mathrm{ER} \mathrm{Ca}^{2+}$ depletion in response to activation of inositol $(1,4,5)$-trisphosphate receptors and $\mathrm{Ca}^{2+}$ release from the ER. ER Ca ${ }^{2+}$ concentration is sensed by helix-loop-helix structural domain (EF hand domain) in the luminal portion of STIM1. Following $\mathrm{Ca}^{2+}$ depletion from the ER, STIM1 forms oligomers and translocates to ER-PM junctions [1]. Once located to ER-PM junctions, STIM1 binds to Orai proteins and activate SOC Ca ${ }^{2+}$ influx, leading to an increase in intracellular $\mathrm{Ca}^{2+}$ concentration. This cytoplasmic $\mathrm{Ca}^{2+}$ abundance is sequestered by SERCA pump to the ER, refilling the stores. The EF hand domain of STIM2 proteins has significantly lower affinity for $\mathrm{Ca}^{2+}$ than STIM1, and it has been established that STIM2 proteins primarily control steady-state ER and cytosolic $\mathrm{Ca}^{2+}$ homeostasis [4].

Both STIM1 and STIM2 proteins are expressed in hippocampal neurons [5], and both of these proteins have been implicated in control of nSOC. Klejman et al. observed that STIM1 is colocalized with Orail upon depletion of $\mathrm{Ca}^{2+}$ stores in dendrites and soma [6]. More recent reports suggested that STIM1 and Orail are colocalized and may interact with synaptopodin, a protein that regulates synaptic plasticity in hippocampal dendritic spines [7]. Activation of STIM1 and nSOC was described in hippocampal neurons following activation of type I metabotropic glutamate receptors (mGluR) or muscarinic acetylcholine receptors (mAChR) [8]. Notably, nSOC-independent functions of STIM1 in hipocampal neurons were also reported. It was suggested that STIM1 directly controls level of phosphorylation and surface expression of the AMPAR [9]. The role of STIM1 in control of neuronal L-type $\mathrm{Ca}^{2+}$ channel activity [10] and feedback regulation of $\mathrm{Ca}^{2+}$ signals in presynaptic terminals was reported [11]. Using optically controlled construct OptoSTIM1, Kyung et al. demonstrated that expression and activation of

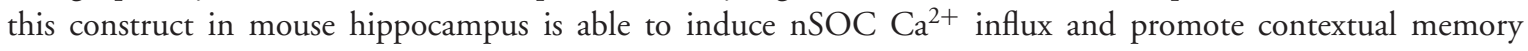
formation [12]. Majewski et al. observed improvements of contextual learning in STIM1 overexpressing mice [13]. These authors further demonstrated that overexpression of STIM1 does not influence long-term potentiaton (LTP) induction in acute hippocampal slices, however, blocks DHPG-dependent long-term depression (LTD) [13]. The mice with forebrain-specific knockout of STIM1 and STIM2 were analyzed by Garcia-Alvarez et al. [14]. These authors reported a mild learning delay in Stim 1 cKO mice, while learning and memory in Stim2 cKO mice was 
normal. However, deletion of both Stim genes resulted in a pronounced impairment in spatial learning and memory and in enhanced LTP [14].

STIM2 is a predominant isoform in the hippocampus [5,15]. Genetic deletion of STIM2 protein impaired SOC and confers protection from neurological damage in a model of focal cerebral ischemia [16]. A recent study by Korkotian et al. suggested that STIM2 moves to dendritic spines and regulates Orai1-mediated nSOC [17]. It has been reported that STIM2, but not STIM1, influences the formation of excitatory dendritic spines and shapes basal synaptic transmission in excitatory neurons $[9,18]$. These authors concluded that STIM2 functions in an nSOC-independent manner by regulating phosphorylation and surface expression of the AMPAR [9]. The same group reported that STIM2 is required for the stable expression of both LTP and LTD at CA3-CA1 hippocampal synapses [18]. Our laboratory reported that virus-mediated knockdown of STIM2 protein expression causes mushroom spine shrinkage and causes loss of nSOC in hippocampal dendritic spines [19]. Moreover, it has been observed that STIM2 hyperexpression as well as pharmacological activation of nSOC in the hippocampus is able to protect mushroom spines in different models of Alzheimer's disease (AD) pathology [15,19-20]. Downregulation of STIM2 proteins was observed in cells from AD patients and in AD mouse models [19,21]. Based on these results we suggested that nSOC-mediated $\mathrm{Ca}^{2+}$ influx is needed to maintain continuous activity of CaMKII in the spines, supporting stability of mushroom spines [19,22].

As it is clear from this brief summary, we are still trying to understand the roles of STIM1 and STIM2 proteins in neurons. Some groups suggest that in neurons STIM1 and STIM2 proteins function in SOC-independent manner [9-11]. Other groups concluded that similar to nonexcitable cells, STIM1 and STIM2 proteins mediate nSOC. It is possible that STIM1 and STIM2 play both nSOC-related and unrelated roles in neurons. Observed discrepancies are likely to be caused by differences in experimental models and protocols. Nevertheless, learning and synaptic plasticity phenotypes observed in STIM1 and STIM2 knockout and overexpression models [12-14,16] suggest that these proteins clearly play a role in neuronal function. It is likely that the main role of nSOC in neurons is signaling and not just refilling of the stores [23]. Future studies will further clarify roles of STIM1 and STIM2 proteins in neuronal physiology.

Acknowledgements

We thank Dr P Plotnikova for administrative support.

Financial \& competing interests disclosure

I Bezprozvanny is a holder of the Carl J and Hortense M Thomsen Chair in Alzheimer's disease research. This work was supported by the Russian Science Foundation Grant 14-25-00024-П (IB; part of the work devoted to the STIM1 role in nSOC regulation), and by the grant of President of Russian Federation 14.Y30.17.1043-MK (EP; part of the work devoted to the STIM2 role in nSOC regulation). The authors have no other relevant affiliations or financial involvement with any organization or entity with a financial interest in or financial conflict with the subject matter or materials discussed in the manuscript apart from those disclosed.

No writing assistance was utilized in the production of this manuscript.

\section{References}

1. Hogan PG, Lewis RS, Rao A. Molecular basis of calcium signaling in lymphocytes: STIM and ORAI. Annu. Rev. Immunol. 28, 491-533 (2010).

2. Lu B, Fivaz M. Neuronal SOCE: myth or reality? Trends Cell Biol. 26(12), 890-893 (2016).

3. Collins SR, Meyer T. Evolutionary origins of STIM1 and STIM2 within ancient $\mathrm{Ca}(2+)$ signaling systems. Trends Cell Biol. 21(4), 202-211 (2011).

4. Brandman O, Liou J, Park WS, Meyer T. STIM2 is a feedback regulator that stabilizes basal cytosolic and endoplasmic reticulum Ca2+ levels. Cell 131(7), 1327-1339 (2007).

5. Gruszczynska-Biegala J, Pomorski P, Wisniewska MB, Kuznicki J. Differential roles for STIM1 and STIM2 in store-operated calcium entry in rat neurons. PloS ONE 6(4), e19285 (2011).

6. Klejman ME, Gruszczynska-Biegala J, Skibinska-Kijek A et al. Expression of STIM1 in brain and puncta-like co-localization of STIM1 and ORAI1 upon depletion of $\mathrm{Ca}(2+)$ store in neurons. Neurochem. Int. 54(1), 49-55 (2009).

7. Vlachos A, Korkotian E, Schonfeld E, Copanaki E, Deller T, Segal M. Synaptopodin regulates plasticity of dendritic spines in hippocampal neurons. J. Neurosci. 29(4), 1017-1033 (2009).

8. Ng AN, Krogh M, Toresson H. Dendritic EGFP-STIM1 activation after type I metabotropic glutamate and muscarinic acetylcholine receptor stimulation in hippocampal neuron. J. Neurosci. Res. 89(8), 1235-1244 (2011). 
9. Garcia-Alvarez G, Lu B, Yap KA et al. STIM2 regulates PKA-dependent phosphorylation and trafficking of AMPARs. Mol. Biol. Cell 26(6), 1141-1159 (2015).

10. Dittmer PJ, Wild AR, Dell'Acqua ML, Sather WA. STIM1 Ca2+ sensor control of L-type Ca2+-channel-dependent dendritic spine structural plasticity and nuclear signaling. Cell. Rep. 19(2), 321-334 (2017).

11. de Juan-Sanz J, Holt GT, Schreiter ER, de Juan F, Kim DS, Ryan TA. Axonal endoplasmic reticulum Ca2+ content controls release probability in CNS nerve terminals. Neuron 93(4), 867-881 (2017).

12. Kyung T, Lee S, Kim JE et al. Optogenetic control of endogenous Ca(2+) channels in vivo. Nat. Biotechnol. 33(10), 1092-1096 (2015).

13. Majewski Ł, Maciąg F, Boguszewski PM et al. Overexpression of STIM1 in neurons in mouse brain improves contextual learning and impairs long-term depression. Biochim. Biophys. Acta 1864(6), 1071-1087 (2017).

14. Garcia-Alvarez G, Shetty MS, Lu B et al. Impaired spatial memory and enhanced long-term potentiation in mice with forebrain-specific ablation of the Stim genes. Front. Behav. Neurosci. 9, 180 (2015).

15. Zhang H, Sun S, Wu L et al. Store-operated calcium channel complex in postsynaptic spines: a new therapeutic target for Alzheimer's disease treatment. J. Neurosci. 36(47), 11837-11850 (2016).

16. Berna-Erro A, Braun A, Kraft R et al. STIM2 regulates capacitive Ca2+ entry in neurons and plays a key role in hypoxic neuronal cell death. Sci. Signal 2(93), ra67 (2009).

17. Korkotian E, Oni-Biton E, Segal M. The role of the store-operated calcium entry channel Orai1 in cultured rat hippocampal synapse formation and plasticity. J. Physiol. 595(1), 125-140 (2017).

18. Yap KA, Shetty MS, Garcia-Alvarez G et al. STIM2 regulates AMPA receptor trafficking and plasticity at hippocampal synapses. Neurobiol. Learn. Mem. 138, 54-61 (2016).

19. Sun S, Zhang H, Liu J et al. Reduced synaptic STIM2 expression and impaired store-operated calcium entry cause destabilization of mature spines in mutant presenilin mice. Neuron 82(1), 79-93 (2014).

20. Popugaeva E, Pchitskaya E, Speshilova A et al. STIM2 protects hippocampal mushroom spines from amyloid synaptotoxicity. Mol. Neurodegener. 10(1), 37 (2015).

21. Bojarski L, Pomorski P, Szybinska A et al. Presenilin-dependent expression of STIM proteins and dysregulation of capacitative Ca2+ entry in familial Alzheimer's disease. Biochim. Biophys. Acta 1793(6), 1050-1057 (2009).

22. Popugaeva E, Vlasova OL, Bezprozvanny I. Restoring calcium homeostasis to treat Alzheimer's disease: a future perspective. Neurodegener. Dis. Manag. 5(5), 395-398 (2015).

23. Majewski L, Kuznicki J. SOCE in neurons: signaling or just refilling? Biochim. Biophys. Acta 1853(9), 1940-1952 (2015). 
